\title{
Asymmetric Reactions of 8-Phenylmenthyl Pyruvate with Allyltrimethylsilane, Silyl Enol Ethers and Ketene Silyl Acetals
}

\author{
Ming-Yi Chen and Jim-Min Fang* \\ Department of Chemistry, National Taiwan University, Taipei, Taiwan 10617, Republic of China
}

\begin{abstract}
By mediation of $\mathrm{TiCl}_{4}$, allyisilane, silyl enol ethers and ketene silyl acetals attacked (-)phenylmenthyl pyruvate and (-)-phenylmenthyl phenylglyoxylate at their si-faces. The reactions are hypothesised to proceed with rigid cyclic transition states: anti aldol adducts 16a and 17a were favourably obtained from $E$-ketene silyl acetals 6 and 7 having $E$-configuration, whereas syn aldol adducts 18b-21b were predominantly obtained from $Z$-ketene silyl acetal 18 and $Z$-silyl enol ethers $19-21$.
\end{abstract}

Formation of $\mathrm{C}-\mathrm{C}$ bonds in a stereoselective controlled manner is an important synthetic method of current interest. Among many chiral auxiliaries, ${ }^{1}$ (-)-8-phenylmenthol is a useful reagent utilized frequently to direct asymmetric reactions of its preformed carboxylates, such as alkylations, ${ }^{2}$ Michael reactions, ${ }^{3}$ Diels-Alder reactions, ${ }^{4}$ Wittig rearrangements ${ }^{5}$ and radical reactions. ${ }^{6}$ Application of (-)-phenylmenthol to asymmetric reactions of $\alpha$-oxocarboxylates has been reported, such as reductions, ${ }^{7}$ additions with Grignard reagents, ${ }^{8}$ additions with crotyltributylstannane or crotyltrimethylsilane mediated by $\mathrm{BF}_{3},{ }^{9}$ the addition with methyl(triisopropoxy)titanium, ${ }^{10}$ additions with alkynyllithium reagents, ${ }^{11}$ ene reactions promoted by $\mathrm{SnCl}_{4},{ }^{12}$ Henry reactions with nitroalkanes $^{13}$ and photochemical $[2+2]$ cycloadditions with oxazolines. ${ }^{14}$ The degree of diastereoselectivity is found to be affected by the counterion of the nucleophile in addition to other controlling factors. In one report, ${ }^{9 b}$ stereochemical addition of crotyltrimethylsilane to (-)-phenylmenthyl pyruvate 1 has been successfully carried out by promotion with $\mathrm{BF}_{3}$ but failed in the presence of $\mathrm{TiCl}_{4}$. However, considering the high stereoselectivities in many examples of $\mathrm{TiCl}_{4}$-mediated nucleophilic reactions toward $\alpha$-oxycarbonyl compounds, ${ }^{15}$ we investigated the reactions of (-)-8-phenylmenthyl pyruvate with allyltrimethylsilane, silyl enol ethers and ketene silyl acetals using the Mukaiyama procedure. These reactions proceeded smoothly and stereoselectively and provided a route to assemble chiral quaternary carbon centres.

\section{Results and Discussion}

The addition of allysilane to (-)-8-phenylmenthyl pyruvate 1 was conducted by mediation of $\mathrm{TiCl}_{4}$ at $-78^{\circ} \mathrm{C}$ in $\mathrm{CH}_{2} \mathrm{Cl}_{2}$ solution (Scheme 1). The reaction gave a single product,<smiles>C=CCC(C)(O)C(=O)OC1CC(C)CC(C(C)C)C1C(C)(C)c1ccccc1</smiles>

Scheme 1 Reagents and conditions: $\mathrm{i}, \mathrm{TiCl}_{4}, \mathrm{CH}_{2} \mathrm{Cl}_{2},-78^{\circ} \mathrm{C}(96 \%)$

the tertiary alcohol 12 having the $2 S$-configuration. The absolute configuration of 12 was confirmed by saponification to $(S)$-( + )-2-hydroxy-2-methylpent-4-enoic acid. ${ }^{16}$

The aldol reactions of 1 with a variety of silyl enol ethers $(3,4$ and 9-11) and ketene silyl acetals (5-8) were carried out by similar procedures. The results are listed in Table 1 . The aldol reactions occurred exclusively at the $s i$-face of the pyruvate to give the products 13-21 of $2 S$-configuration. Depending on the configuration of the silyl enol ether or ketene silyl acetal, the aldol reaction showed asymmetric induction to give either the 2,3-syn or 2,3-anti product as the major product. The ketene silyl acetals 6 and 7 of $Z$-configuration yielded predominantly the anti isomers of 16 and 17 in $2 S, 3 R$ configuration, whereas the ketene silyl acetal 8 and silyl enol ethers 9-11 of $E$ configuration produced the aldol adducts 18-21 in predominantly $2 S, 3 S$ configuration.

The additions using the 8-phenylmenthol auxiliary appeared to exert better stereocontrol than the corresponding reactions of 2-4 with (-)-menthyl pyruvate [44-55\% diastereoisomeric excess (d.e.) ]. ${ }^{17}$ The reaction of ketene silyl acetal 5 with (-)-8phenylmenthyl phenylglyoxylate in the presence of $\mathrm{TiCl}_{4}$ gave the aldol adduct 22 exclusively in the $2 R$-configuration. The reaction of 6 under similar conditions also afforded a single product 23 having the $2 R, 3 R$ configuration. 21a $(2 S, 3 R)$<smiles>CC(=O)C(C)(C)C(O)(C(=O)OC1CC(C)CCC1C(C)(C)c1ccccc1)c1ccccc1</smiles>

$22(2 R)$<smiles>CC1CC[C@H](OC(=O)[C@H](C)[C@H]2CC(C(C)(C)C)CCC2=O)C(C(C)(C)c2ccccc2)C1</smiles><smiles>CC1CC[C@H](C(C)(C)c2ccccc2)C(OC(=O)[C@H](C)[C@H]2C[C@@H](C(C)(C)C)CCC2=O)C1</smiles>

$21 \mathrm{~b}(2 S, 3 S)$<smiles>CCC(C(C)=O)C(O)(C(=O)OC1CC(C)CCC1C(C)(C)c1ccccc1)c1ccccc1</smiles>

$23(2 R, 3 R)$
The stereochemical outcome shown in Table 1 implies that the $\mathrm{TiCl}_{4}$-mediated reactions proceeded via rigid transition states as depicted in Fig. 1. ${ }^{17,18}$ The titanium enolate generated from 3-11 was chelated with both carbonyl groups of the pyruvate, the phenyl group of the auxiliary was preferably oriented to the endo face of the pyruvate, and the aldol reaction occurred at the less hindered face (si-face) of (-)-8-phenylmenthyl pyruvate via a [3.2.1] bicyclic transition state to give the observed major products. This model explains the consequence that the $2 S, 3 R$-adduct is obtained from the $Z$-enolate and the $2 S, 3 S$-adduct from the $E$-enolate. The products $21 \mathrm{a}$ and $21 \mathrm{~b}$ 
Table 1 Reactions of (-)-8-phenylmenthyl pyruvate with silyl enol ethers and ketene silyl acetals $\left(\mathrm{TiCl}_{4}, \mathrm{CH}_{2} \mathrm{Cl}_{2},-78^{\circ} \mathrm{C}, 10 \mathrm{~min}\right)$

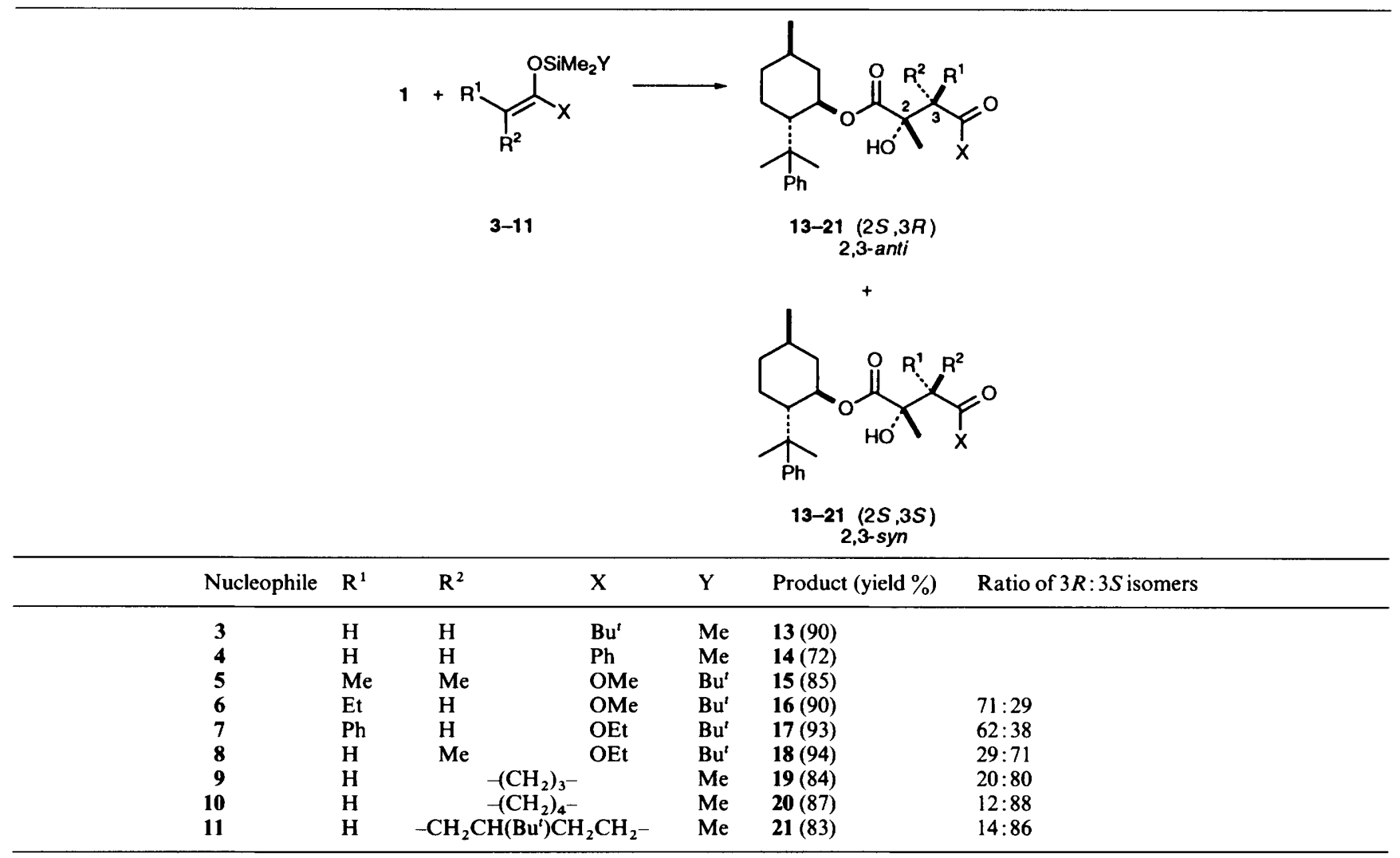

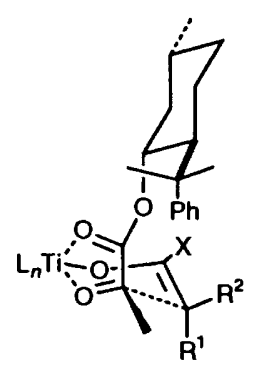

Fig. 1

were obtained presumably by approaching the silyl enol ether of 4-tert-butylcyclohexanone 11 at the equatorial position.

\section{Experimental}

General information concerning instrumentation and materials was described previously. ${ }^{19}(-)-8$-Phenylmenthyl pyruvate and (-)-8-phenylmenthyl phenylglyoxylate were prepared ${ }^{20}$ by acid-catalysed condensation of $(1 R, 2 S, 5 R)-(-)-8$-phenylmenthol ${ }^{21}$ with pyruvic acid and benzoylformic acid, respectively. The silyl enol ethers and ketene silyl acetals 3-11 were prepared according to literature methods. ${ }^{22}[\alpha]_{\mathrm{D}}$ values are given in units of $10^{-1} \mathrm{deg} \mathrm{cm}^{2} \mathrm{~g}^{-1} . J$ Values are given in $\mathrm{Hz}$.

General Procedure for Reactions of (-)-8-Phenylmenthyl Pyruvate with Allyltrimethylsilane, Silyl Enol Ethers and Ketene Silyl Acetals 2-11. - Under an atmosphere of $\mathrm{N}_{2}, \mathrm{TiCl}_{4}(0.07$ $\left.\mathrm{cm}^{3}, 0.6 \mathrm{mmol}\right)$ was added dropwise to a cold $\left(-78^{\circ} \mathrm{C}\right)$ stirred solution of (-)-8-phenylmenthyl pyruvate $(0.5 \mathrm{mmol})$ in anhydrous $\mathrm{CH}_{2} \mathrm{Cl}_{2}$. A solution of the appropriate nucleophile (allyltrimethylsilane, silyl enol ether or ketene silyl acetal, 0.6 mmol) was subsequently added dropwise. The reaction mixture was stirred for $20 \mathrm{~min}$ at $-78^{\circ} \mathrm{C}$ and poured into ice-water.
After removal of $\mathrm{CH}_{2} \mathrm{Cl}_{2}$ by rotary evaporator, the residue was extracted three times with EtOAc. The combined extracts were washed with brine, dried $\left(\mathrm{Na}_{2} \mathrm{SO}_{4}\right)$ and then concentrated under reduced pressure to give crude products 12-21. Separation of products was accomplished by column chromatography $\left(\mathrm{SiO}_{2}\right)$ and/or HPLC with elution of the gradients of EtOAc in hexane indicated in each case. The reactions of $(-)$ phenylmenthyl phenylglyoxylate with ketene silyl acetals 5 and 6 were carried out by similar procedures to give 22 and 23 , respectively, in 80 and $78 \%$ yields.

5-Methyl-2-(1-methyl-1-phenylethyl)cyclohexyl 2-Hydroxy2-methylpent-4-enoate 12.-2S-Isomer: oil; HPLC (3\% EtOAc in hexane) $t_{\mathrm{R}} 14.3 \mathrm{~min} ;[\alpha]_{\mathrm{D}}^{25}-8.6\left(\mathrm{CHCl}_{3}, c 7\right) ; v_{\max }$ (neat)/ $\mathrm{cm}^{-1} 3516,1714$ and $1595 ; \delta_{\mathrm{H}}\left(\mathrm{CDCl}_{3}, 200 \mathrm{MHz}\right) 0.72-1.14$ ( $2 \mathrm{H}, \mathrm{m}), 0.88$ (3 H, d, J 6.4, Me), 1.16 (3 H, s, Me), 1.17-1.29 $(2 \mathrm{H}, \mathrm{m}), 1.22(3 \mathrm{H}, \mathrm{s}, \mathrm{Me}), 1.32(3 \mathrm{H}, \mathrm{s}, \mathrm{Me}), 1.36-1.64(2 \mathrm{H}, \mathrm{m})$, 1.94 $(1 \mathrm{H}, \mathrm{m}), 2.09(1 \mathrm{H}$, ddd, $J 10.7,10.7,5.5), 2.30(2 \mathrm{H}, \mathrm{m}), 2.65$ (1 H, br s, OH), 4.88 (1 H, ddd, $J 10.6,10.6,4.4), 5.04-5.14(2 \mathrm{H}$, $\mathrm{m}), 5.64-5.84(1 \mathrm{H}, \mathrm{m}), 7.11-7.24(1 \mathrm{H}, \mathrm{m}, \mathrm{PhH})$ and 7.28-7.40 $(4 \mathrm{H}, \mathrm{m}, \mathrm{PhH}) ; \delta_{\mathrm{C}}\left(\mathrm{CDCl}_{3}, 50 \mathrm{MHz}\right) 21.6(\mathrm{q}), 24.5(\mathrm{q}), 26.6(\mathrm{q})$, $26.8(\mathrm{q}), 27.0(\mathrm{t}), 31.2(\mathrm{~d}), 34.3(\mathrm{t}), 39.6(\mathrm{~s}), 41.5(\mathrm{t}), 44.3(\mathrm{~d}), 49.6$ (d), 73.8(s), 76.8(d), 118.9(t), 125.2(d), 125.3(d), 125.6(d), 128.1 (d), 128.3 (d), 132.3 (d), 151.0 (s) and $175.5\left(\mathrm{~s}, \mathrm{CO}_{2}\right) ; \mathrm{m} / \mathrm{z} 344$ $\left(\mathrm{M}^{+}, 13 \%\right)$ and 119 (100) (Found: $\mathrm{M}^{+}, 344.2344$. Calc. for $\mathrm{C}_{22} \mathrm{H}_{32} \mathrm{O}_{3}, M, 344.2351$ ). Saponification of 12 with aqueous $\mathrm{KOH}$ in $\mathrm{MeOH}$ gave $(S)$-2-hydroxy-2-methylpent-4-enoic acid, viz. citramalic acid, as an oil; $[\alpha]_{\mathrm{D}}^{25}+10.6\left(\mathrm{CHCl}_{3}, c 9.2\right)$, lit., ${ }^{16}[\alpha]_{\mathrm{D}}^{25}+11.3(\mathrm{EtOH} c 1) ; \delta_{\mathrm{C}}\left(\mathrm{CDCl}_{3}\right) 25.3(\mathrm{q}), 44.3(\mathrm{t})$, $74.5(\mathrm{~s}), 119.9(\mathrm{t}), 131.7(\mathrm{~d})$ and $209.3(\mathrm{~s})$.

5-Methyl-2-(1-methyl-1-phenylethyl)cyclohexyl 2-Hydroxy2,5,5-trimethyl-4-oxohexanoate 13.- $2 S$-Isomer: oil; TLC $(5 \%$ EtOAc in hexane) $R_{\mathrm{f}} 0.12 ;[\alpha]_{\mathrm{D}}^{25}+17.8\left(\mathrm{CHCl}_{3}, c \quad 6.6\right)$; $v_{\max }($ neat $) / \mathrm{cm}^{-1} 3543,1730$ and $1600 ; \delta_{\mathrm{H}}\left(\mathrm{CDCl}_{3}\right) 0.73-1.05(3 \mathrm{H}$, 
m), $0.87(3 \mathrm{H}, \mathrm{d}, J 6.4), 1.14(9 \mathrm{H}, \mathrm{s}), 1.20(3 \mathrm{H}, \mathrm{s}), 1.25(3 \mathrm{H}, \mathrm{s})$, $1.31-1.56(2 \mathrm{H}, \mathrm{m}), 1.90(3 \mathrm{H}, \mathrm{s}), 2.00-2.06(2 \mathrm{H}, \mathrm{m}), 2.64(1 \mathrm{H}, \mathrm{d}$, $J 17.8), 2.93(1 \mathrm{H}, \mathrm{d}, J 17.8), 4.79(1 \mathrm{H}$, ddd, $J 10.4,10.4,4.6)$, 7.10-7.17 $(1 \mathrm{H}, \mathrm{m})$ and 7.19-7.32 $(4 \mathrm{H}, \mathrm{m}) ; \delta_{\mathrm{C}}\left(\mathrm{CDCl}_{3}\right) 21.6(\mathrm{q})$, 25.6 (q, 3 C), 26.2 (q), $26.6(\mathrm{q}), 26.9$ (q), 30.1 (s), 31.1 (t), 34.4 (d), $39.7(\mathrm{t}), 40.9(\mathrm{~s}), 43.7(\mathrm{t}), 45.7(\mathrm{t}), 49.7(\mathrm{~d}), 72.3(\mathrm{~s}), 76.7(\mathrm{~d})$, 125.1 (d), 125.5 (d, 2 C), 127.9 (d, 2 C), $151.0(\mathrm{~s}), 174.9\left(\mathrm{~s}, \mathrm{CO}_{2}\right)$ and $214.8(\mathrm{~S}, \mathrm{CO}) ; m / z 402\left(\mathrm{M}^{+}, 6 \%\right)$ and $119(100)$ (Found: $\mathrm{M}^{+}, 402.2761$. Calc. for $\left.\mathrm{C}_{25} \mathrm{H}_{38} \mathrm{O}_{4}, M, 402.2770\right)$.

5-Methyl-2-(1-methyl-1-phenylethyl)cyclohexyl 2-Hydroxy2-methyl-4-oxo-4-phenylbutanoate 14.-2S-Isomer: oil; TLC (10\% EtOAc in hexane) $R_{\mathrm{f}} 0.24 ;[\alpha]_{\mathrm{D}}^{25}+29.9\left(\mathrm{CHCl}_{3}, c 1.2\right)$; $v_{\max }$ (neat) $/ \mathrm{cm}^{-1} 3543$ and $1728 ; \delta_{\mathrm{H}}\left(\mathrm{CDCl}_{3}\right) 0.69-1.51(3 \mathrm{H}, \mathrm{m})$, $0.84(3 \mathrm{H}, \mathrm{d}, J 6.4), 1.24(3 \mathrm{H}, \mathrm{s}), 1.31(3 \mathrm{H}, \mathrm{s}), 1.42(3 \mathrm{H}, \mathrm{s}), 1.35-$ $1.52(3 \mathrm{H}, \mathrm{m}), 1.96-2.13(2 \mathrm{H}, \mathrm{m}), 3.09(1 \mathrm{H}, \mathrm{d}, J 18.0), 3.32(1 \mathrm{H}$, d, $J 18.0), 3.71(1 \mathrm{H}, \mathrm{br} \mathrm{s}, \mathrm{OH}), 4.85(1 \mathrm{H}$, ddd, $J 10.6,10.6,4.5)$, 7.11-7.38 (5 H, m), 7.41-7.52 (3 H, m) and 7.87-7.93 (2 H, m); $\delta_{\mathrm{C}}\left(\mathrm{CDCl}_{3}\right) 21.7(\mathrm{q}), 25.9(\mathrm{q}), 26.8(\mathrm{q}), 27.0(\mathrm{q}), 29.8(\mathrm{t}), 31.2(\mathrm{~d})$, $34.5(\mathrm{~d}), 39.9(\mathrm{~s}), 40.8(\mathrm{t}), 47.3(\mathrm{t}), 49.7(\mathrm{~d}), 72.7(\mathrm{~s}), 77.0(\mathrm{~d}), 125.2$ (d, 2 C), 125.6(d, 2 C), $128.0(\mathrm{~d}, 2 \mathrm{C}), 128.1(\mathrm{~d}, 2 \mathrm{C}), 128.6(\mathrm{~d}, 2 \mathrm{C})$, $133.5(\mathrm{~s}), 151.4(\mathrm{~s}), 175.0(\mathrm{~s})$ and $198.7(\mathrm{~s}) ; m / z 422\left(\mathrm{M}^{+}, 8 \%\right), 119$ (100) (Found: $\mathrm{M}^{+}, 422.2433$. Calc. for $\mathrm{C}_{27} \mathrm{H}_{34} \mathrm{O}_{4}, M, 422.2457$ ).

1-[5-Methyl-2-(1-methyl-1-phenylethyl)cyclohexyl] 4-Methyl 2-Hydroxy-2,3,3-trimethylbutanedioate 15.-2S-Isomer: oil TLC $\left(10 \%\right.$ EtOAc in hexane) $R_{\mathrm{f}} 0.20 ;[\alpha]_{\mathrm{D}}^{25}-40.6\left(\mathrm{CHCl}_{3}, c\right.$ $0.4) ; v_{\max }($ neat $) / \mathrm{cm}^{-1} 3511$ and $1727 ; \delta_{\mathrm{H}}\left(\mathrm{CDCl}_{3}\right) 0.70-1.23(3 \mathrm{H}$, $\mathrm{m}), 0.87(3 \mathrm{H}, \mathrm{d}, J 6.4), 1.27(12 \mathrm{H}, \mathrm{br} \mathrm{s}), 1.30-1.62(3 \mathrm{H}, \mathrm{m}), 1.37$ $(3 \mathrm{H}, \mathrm{s}), 1.95-2.10(2 \mathrm{H}, \mathrm{m}), 3.72(3 \mathrm{H}, \mathrm{s}), 4.94(1 \mathrm{H}$, ddd, $J 10.6$, $10.6,4.6), 7.11-7.22(1 \mathrm{H}, \mathrm{m})$ and $7.24-7.35(4 \mathrm{H}, \mathrm{m}) ; \delta_{\mathrm{C}}\left(\mathrm{CDCl}_{3}\right)$ $20.3(\mathrm{q}), 21.2(\mathrm{q}), 21.6(\mathrm{q}), 21.7(\mathrm{q}), 25.1$ (q), $27.2(\mathrm{t}), 29.0(\mathrm{q}), 31.3$ (d), 34.3(t), 40.1 (s), 41.3(t), 49.2(s), 50.0 (d), 52.0 (t), 77.6 (d), $77.6(\mathrm{~s}), 125.4(\mathrm{~d}), 125.5(\mathrm{~d}, 2 \mathrm{C}), 128.1(\mathrm{~d}, 2 \mathrm{C}), 150.5(\mathrm{~s}), 175.0(\mathrm{~s})$ and $176.6(\mathrm{~s}) ; \mathrm{m} / \mathrm{z} 404\left(\mathrm{M}^{+}, 1 \%\right)$ and $119(100)$ (Found: $\mathrm{M}^{+}$, 404.2552. Calc. for $\mathrm{C}_{24} \mathrm{H}_{36} \mathrm{O}_{5}, M, 404.2563$ ).

1-[5-Methyl-2-(1-methyl-1-phenylethyl)cyclohexyl] 4-Methyl 3-Ethyl-2-hydroxy-2-methylbutanedioate 16-2S,3R-Isomer:oil; TLC ( $3 \%$ EtOAc in hexane) $R_{\mathrm{f}} 0.08 ;[\alpha]_{\mathrm{D}}^{25}-5.7\left(\mathrm{CHCl}_{3}, c 1.2\right)$; $v_{\max }($ neat $) / \mathrm{cm}^{-1} 3526$ and $1734 ; \delta_{\mathrm{H}}\left(\mathrm{CDCl}_{3}\right) 0.84-1.12(3 \mathrm{H}, \mathrm{m})$, $0.87(3 \mathrm{H}, \mathrm{d}, J 6.4 \mathrm{~Hz}), 0.88(3 \mathrm{H}, \mathrm{t}, J 7.4), 1.18(3 \mathrm{H}, \mathrm{s}), 1.24(3 \mathrm{H})$, $1.34(3 \mathrm{H}, \mathrm{s}), 1.35-1.77(3 \mathrm{H}, \mathrm{m}), 1.93(1 \mathrm{H}, \mathrm{m}), 2.07(1 \mathrm{H}, \mathrm{ddd}, J$ $10.1,10.1,4.4), 2.52(1 \mathrm{H}, \mathrm{dd}, J 6.4,4.5), 3.68(3 \mathrm{H}, \mathrm{s}), 4.86(1 \mathrm{H}$, ddd, $J 10.6,10.6,4.4), 7.15-7.20(1 \mathrm{H}, \mathrm{m})$ and $7.25-7.32(4 \mathrm{H}, \mathrm{m})$; $\delta_{\mathrm{C}}\left(\mathrm{CDCl}_{3}\right) 12.3(\mathrm{q}), 19.7(\mathrm{q}), 21.8(\mathrm{q}), 22.3(\mathrm{q}), 26.0(\mathrm{q}), 27.1(\mathrm{t})$, $28.0(\mathrm{t}), 31.2(\mathrm{~d}), 34.4(\mathrm{t}), 40.0(\mathrm{~s}), 41.0(\mathrm{t}), 49.7(\mathrm{~d}), 51.4(\mathrm{~d}), 53.1$ (d), 75.0(s), 77.0 (d), 125.3 (d), 125.6(d, 2 C), 128.1 (d, 2C), 150.8 (s), $175.0(\mathrm{~s})$ and $175.1(\mathrm{~s}) ; \mathrm{m} / \mathrm{z} 404\left(\mathrm{M}^{+}, 7 \%\right)$ and $119(100)$ (Found: $\mathrm{M}^{+}$, 404.2541. Calc. for $\mathrm{C}_{24} \mathrm{H}_{36} \mathrm{O}_{5}, M, 404.2563$ ). $2 S, 3 S$-Isomer: oil; TLC ( $3 \%$ EtOAc in hexane) $R_{\mathrm{f}} 0.09$; $[\alpha]_{\mathrm{D}}^{25}$ $+0.1 \quad\left(\mathrm{CHCl}_{3}, \quad c \quad 3.7\right) ; \quad v_{\max }($ neat $) / \mathrm{cm}^{-1} 3495$ and 1733; $\delta_{\mathrm{H}}\left(\mathrm{CDCl}_{3}\right) 0.85-1.16(3 \mathrm{H}, \mathrm{m}), 0.88(3 \mathrm{H}, \mathrm{d}, J 6.4), 0.89(3 \mathrm{H}, \mathrm{t}$, J7.4), $1.21(3 \mathrm{H}, \mathrm{s}), 1.23(3 \mathrm{H}, \mathrm{s}), 1.34(3 \mathrm{H}, \mathrm{s}), 1.38-1.63(3 \mathrm{H}, \mathrm{m})$, $1.80(1 \mathrm{H}, \mathrm{m}), 1.98(1 \mathrm{H}, \mathrm{m}), 2.10(1 \mathrm{H}, \mathrm{ddd}, J 10.0,10.0,3.9), 2.54$ ( $1 \mathrm{H}$, dd, $J 12.0,3.1$ ), $3.71(3 \mathrm{H}, \mathrm{s}), 4.91$ (1 H, ddd, $J$ 10.6, 10.6, 4.4), 7.15-7.20 (1 H, m) and 7.25-7.31 (4 H, m); $\delta_{\mathrm{C}}\left(\mathrm{CDCl}_{3}\right)$ $12.3(\mathrm{q}), 20.8(\mathrm{q}), 21.7(\mathrm{q}), 23.5(\mathrm{q}), 26.2(\mathrm{q}), 27.0(\mathrm{t}), 27.5(\mathrm{t})$, 31.2 (d), 34.3 (t), 39.9 (s), 41.1 (t), 49.7 (d), 51.5 (d), 55.6 (d), 75.0 (s), 77.5 (d), 125.4 (d, 3 C), 128.1 (d, 2 C), 150.8 (s) $173.5(\mathrm{~s})$ and $174.3(\mathrm{~s}) ; m / z 405\left(\mathrm{M}^{+}+1,7 \%\right) 119$ (100) (Found: $\mathrm{M}^{+}$, 404.2566. Calc. for $\left.\mathrm{C}_{24} \mathrm{H}_{36} \mathrm{O}_{5}, M, 404.2563\right)$.

1-[5-Methyl-2-(1-methyl-1-phenylethyl)cyclohexyl] 4-Ethyl 2-Hydroxy-2-methyl-3-phenylbutanedioate 17.-2S,3R-Isomer: solid, m.p. $109-110^{\circ} \mathrm{C}$; HPLC (15\% EtOAc in hexane) $t_{\mathrm{R}} 4.8$ $\min ;[\alpha]_{\mathrm{D}}^{25}+14.5\left(\mathrm{CHCl}_{3}, c 8\right) ; v_{\max }(\mathrm{KBr}) / \mathrm{cm}^{-1} 3540$ and 1736 ; $\delta_{\mathrm{H}}\left(\mathrm{CDCl}_{3}\right) 0.75-1.13(3 \mathrm{H}, \mathrm{m}), 0.89(3 \mathrm{H}, \mathrm{d}, J 6.4), 1.19(3 \mathrm{H}, \mathrm{d}, J$
7.4), $1.26(3 \mathrm{H}, \mathrm{s}), 1.40(3 \mathrm{H}, \mathrm{s}), 1.41-1.58(3 \mathrm{H}, \mathrm{m}), 2.02(1 \mathrm{H}, \mathrm{m})$, $2.14(1 \mathrm{H}, \mathrm{d}, J 10.5,10.5,4.6), 3.83(1 \mathrm{H}, \mathrm{br} \mathrm{s}, \mathrm{OH}), 4.04(1 \mathrm{H}, \mathrm{s})$, 4.93 ( $1 \mathrm{H}$, ddd, $J 10.6,10.6,4.5)$ and $7.14-7.40(10 \mathrm{H}, \mathrm{m})$; $\delta_{\mathrm{C}}\left(\mathrm{CDCl}_{3}\right) 14.0(\mathrm{q}), 21.7(\mathrm{q}), 23.3(\mathrm{q}), 26.3(\mathrm{q}), 27.0(\mathrm{t}), 27.7(\mathrm{q})$, $31.2(\mathrm{~d}), 34.5(\mathrm{t}), 40.0(\mathrm{~s}), 41.1(\mathrm{t}), 49.7(\mathrm{~d}), 56.7(\mathrm{~d}), 61.1(\mathrm{t}), 75.1$ (s), 77.0(d), 125.4(d), 125.6(d,2C), 127.7(d), 128.1 (d, 2C), 128.2 (d, 2 C), 130.1 (d, 2 C), $133.7(\mathrm{~s}), 150.9(\mathrm{~s}), 173.2(\mathrm{~s})$ and $175.2(\mathrm{~s})$; $m / z 405\left(\mathrm{M}^{+}+1,7 \%\right)$ and $119(100)$ (Found: C, 74.3; H, 8.1. $\mathrm{C}_{29} \mathrm{H}_{38} \mathrm{O}_{5}$ requires $\mathrm{C}, 74.28 ; \mathrm{H}, 8.03 \%$ ). $2 S, 3 S$-Isomer: oil; HPLC ( $15 \%$ EtOAc in hexane) $t_{\mathrm{R}} 6.6 \mathrm{~min} ;[\alpha]_{\mathrm{D}}^{25}-21.8\left(\mathrm{CHCl}_{3}\right.$, c 5.5); $v_{\max }(\mathrm{KBr}) / \mathrm{cm}^{-1} 3540$ and $1735 ; \delta_{\mathrm{H}}\left(\mathrm{CDCl}_{3}\right) 0.70-0.97$ $(3 \mathrm{H}, \mathrm{m}), 0.80(3 \mathrm{H}, \mathrm{d}, J 6.4), 1.04(3 \mathrm{H}, \mathrm{s}), 1.10(3 \mathrm{H}, \mathrm{s}), 1.25(3 \mathrm{H}$, $\mathrm{t}, J 7.4), 1.27-1.42(3 \mathrm{H}, \mathrm{m}), 1.40(3 \mathrm{H}, \mathrm{s}), 1.52(1 \mathrm{H}, \mathrm{m}), 1.95(1 \mathrm{H}$, d, $J 10.6,10.6,5.2), 3.53(1 \mathrm{H}$, br s, OH $), 3.97(1 \mathrm{H}, \mathrm{s}), 4.17(2 \mathrm{H}$, $\mathrm{m}), 4.71(1 \mathrm{H}$, ddd, $J 10.6,10.6,4.6), 7.10-7.31(8 \mathrm{H}, \mathrm{m})$ and 7.36 $7.47(2 \mathrm{H}, \mathrm{m}) ; \delta_{\mathrm{C}}\left(\mathrm{CDCl}_{3}\right) 14.0(\mathrm{q}), 21.6(\mathrm{q}), 25.1(\mathrm{q}), 25.5(\mathrm{q}), 27.1$ (t), $28.2(\mathrm{q}), 31.2(\mathrm{~d}), 34.4(\mathrm{t}), 39.8(\mathrm{~s}), 40.9(\mathrm{t}), 49.7(\mathrm{~d}), 57.5(\mathrm{~d})$, $61.1(\mathrm{t}), 76.0(\mathrm{~s}), 77.6(\mathrm{~d}), 125.3(\mathrm{~d}), 125.5(\mathrm{~d}, 2 \mathrm{C}), 127.8(\mathrm{~d}), 128.0$ $(\mathrm{d}, 2 \mathrm{C}), 128.1(\mathrm{~d}, 2 \mathrm{C}), 130.1(\mathrm{~d}, 2 \mathrm{C}), 134.2(\mathrm{~s}), 150.8(\mathrm{~s}), 171.7(\mathrm{~s})$ and $173.3(\mathrm{~s}) ; \mathrm{m} / \mathrm{z} 405\left(\mathrm{M}^{+}+1,5 \%\right)$ and $119(100)$ (Found: $\mathrm{C}$, $74.3 ; \mathrm{H}, 8.1 \%$ ).

1-[5-Methyl-2-(1-methyl-1-phenylethyl)cyclohexyl 4-Ethyl 2Hydroxy-2,3-dimethylbutanedioate 18.-2S,3S-Isomer: oil HPLC $\left(15 \%\right.$ EtOAC in hexane) $t_{\mathrm{R}} 6.6 \mathrm{~min} ;[\alpha]_{\mathrm{D}}^{25}-5.7\left(\mathrm{CHCl}_{3}\right.$, c 3.6); $v_{\max }($ neat $) / \mathrm{cm}^{-1} 3512$ and $1721 ; \delta_{\mathrm{H}}\left(\mathrm{CDCl}_{3}\right) 0.76-1.05$ $(3 \mathrm{H}, \mathrm{m}), 0.86(3 \mathrm{H}, \mathrm{d}, J 6.4), 1.18(3 \mathrm{H}, \mathrm{s}), 1.20(3 \mathrm{H}, \mathrm{d}, J 7.3), 1.23$ $(3 \mathrm{H}, \mathrm{s}), 1.27(3 \mathrm{H}, \mathrm{t}, J 7.2), 1.35(3 \mathrm{H}, \mathrm{s}), 1.44-1.60(3 \mathrm{H}, \mathrm{m}), 1.95$ $2.10(2 \mathrm{H}, \mathrm{m}), 2.68(1 \mathrm{H}, \mathrm{q}, J 7.3), 3.54(1 \mathrm{H}, \mathrm{br} \mathrm{s}, \mathrm{OH}), 4.16(2 \mathrm{H}$, q, J7.2), 4.86 (1 H, ddd, $J 10.6,10.6,4.3), 7.13-7.19(1 \mathrm{H}, \mathrm{m})$ and $7.24-7.33(4 \mathrm{H}) ; \delta_{\mathrm{C}}\left(\mathrm{CDCl}_{3}\right) 11.0(\mathrm{q}), 14.1(\mathrm{q}), 21.7(\mathrm{q}), 22.5(\mathrm{q})$, $25.9(\mathrm{q}), 27.1(\mathrm{q}), 27.9(\mathrm{t}), 31.2(\mathrm{~d}), 34.4(\mathrm{t}), 40.0(\mathrm{~s}), 41.0(\mathrm{t}), 45.4$ (t), 49.8 (d), 60.6 (d), 74.6 (s), 76.9 (d), $125.2(\mathrm{~d}), 125.5$ (d, 2 C), $128.0(\mathrm{~d}, 2 \mathrm{C}), 150.8(\mathrm{~s}), 175.2(\mathrm{~s})$ and $175.3(\mathrm{~s}), \mathrm{m} / z 405\left(\mathrm{M}^{+}+1\right.$, $7 \%$ ) and 119 (100) (Found: $\mathrm{M}^{+}$, 404.2563. Calc. for $\left.\mathrm{C}_{24} \mathrm{H}_{36} \mathrm{O}_{5}, M, 404.2563\right)$. 2S,3R-Isomer: oil; HPLC (15\% EtOAc in hexane) $t_{\mathrm{R}} 7.2 \mathrm{~min} ;[\alpha]_{\mathrm{D}}^{25}-0.05\left(\mathrm{CHCl}_{3}, c 3\right)$ $v_{\max }($ neat $) / \mathrm{cm}^{-1} 3515$ and $1724 ; \delta_{\mathrm{H}}\left(\mathrm{CDCl}_{3}\right) 0.72-1.12(3 \mathrm{H}, \mathrm{m})$, $0.88(3 \mathrm{H}, \mathrm{d}, J 6.4), 1.18(3 \mathrm{H}, \mathrm{d}, J 7.3), 1.25(6 \mathrm{H}, \mathrm{s}), 1.28(3 \mathrm{H}, \mathrm{t}, J$ 7.2), $1.35(3 \mathrm{H}, \mathrm{s}), 1.37-1.62(3 \mathrm{H}, \mathrm{m}), 1.96-2.13(2 \mathrm{H}, \mathrm{m}), 2.72$ (1 H, q, J 7.3), 3.24 (1 H, br s, OH ), 4.17 (2 H, q, J 7.2), 4.92 (1 H, ddd, $J 10.6,10.6,4.3), 7.12-7.22(1 \mathrm{H}, \mathrm{m})$ and $7.26-7.35(4 \mathrm{H}, \mathrm{m})$; $\delta_{\mathrm{C}}\left(\mathrm{CDCl}_{3}\right) 12.6(\mathrm{q}), 14.1(\mathrm{q}), 21.7(\mathrm{q}), 23.6(\mathrm{q}), 25.9(\mathrm{q}), 27.1(\mathrm{q})$, $28.0(\mathrm{t}), 31.3(\mathrm{~d}), 34.4(\mathrm{t}), 40.0(\mathrm{~s}), 41.3(\mathrm{t}), 47.6(\mathrm{t}), 49.8(\mathrm{~d}), 60.6$ (d), 75.1 (s), 77.4(d), 125.4(d), 125.5(d,2 C), 128.1 (d, 2 C), 150.8 (s), $173.7(\mathrm{~s})$ and $174.2(\mathrm{~s}) ; 405\left(\mathrm{M}^{+}+1,7 \%\right)$ and $119(100)$ (Found: $\mathrm{M}^{+}, 404.2560$. Calc. for $\mathrm{C}_{24} \mathrm{H}_{36} \mathrm{O}_{5}, M, 404.2563$ ).

5-Methyl-2-(1-methyl-1-phenylethyl)cyclohexyl 2-Hydroxy2-(2-oxocyclopentyl)propanoate 19.-2S,3R-Isomer: oil; HPLC $\left(15 \%\right.$ EtOAc in hexane) $t_{\mathrm{R}} 9.0 \mathrm{~min} ;[\alpha]_{\mathrm{D}}^{25}-40.6\left(\mathrm{CHCl}_{3}, c 0.4\right)$; $v_{\max }($ neat $) / \mathrm{cm}^{-1} 3513$ and $1739 ; \delta_{\mathrm{H}}\left(\mathrm{CDCl}_{3}\right) 0.85-1.08(3 \mathrm{H}, \mathrm{m})$, $1.11(3 \mathrm{H}, \mathrm{d}, J 6.4), 1.27(3 \mathrm{H}, \mathrm{s}), 1.28(3 \mathrm{H}, \mathrm{s}), 1.35(3 \mathrm{H}, \mathrm{s}), 1.40$ $1.84(4 \mathrm{H}, \mathrm{m}), 1.85-2.19(5 \mathrm{H}, \mathrm{m}), 2.20-2.37(2 \mathrm{H}, \mathrm{m}), 2.47(1 \mathrm{H}$, ddd, $J$ 10.6, 10.6, 5.5), $2.76(1 \mathrm{H}$, br s, OH), $4.85(1 \mathrm{H}$, ddd, $J$ $10.6,10.6,4.4), 7.07-7.16(1 \mathrm{H}, \mathrm{m})$ and $7.19-7.38(4 \mathrm{H}, \mathrm{m})$ $\delta_{\mathrm{C}}\left(\mathrm{CDCl}_{3}\right) 20.5(\mathrm{q}), 21.8(\mathrm{q}), 23.8(\mathrm{q}), 25.5(\mathrm{q}), 25.8(\mathrm{t}), 27.2(\mathrm{t})$, $28.6(\mathrm{t}), 31.4(\mathrm{~d}), 34.4(\mathrm{t}), 39.2(\mathrm{t}), 40.1(\mathrm{~s}), 41.4(\mathrm{t}), 50.1(\mathrm{~d}), 54.5$ (d), 76.2 (s), 77.6 (d), 125.4 (d), 125.6 (d, 2 C), 128.1 (d, 2 C), $150.6(\mathrm{~s}), 174.2(\mathrm{~s})$ and $219.8(\mathrm{~s}) ; m / 2386\left(\mathrm{M}^{+}, 1 \%\right)$ and 119 (100) (Found: $\mathrm{M}^{+}, 386.2611$. Calc. for $\mathrm{C}_{24} \mathrm{H}_{34} \mathrm{O}_{4}, M$, 386.2613). $2 S$,3S-Isomer: solid, m.p. $112-114^{\circ} \mathrm{C}$; HPLC $(15 \%$ EtOAc in hexane) $t_{\mathrm{R}} 9.6 \mathrm{~min} ;[\alpha]_{\mathrm{D}}^{25}+16.2\left(\mathrm{CHCl}_{3}, c 1.5\right)$; $v_{\max }($ neat $) / \mathrm{cm}^{-1} 2960$ and 1735; $\delta_{\mathrm{H}}\left(\mathrm{CDCl}_{3}\right) 0.85-1.06(3 \mathrm{H}, \mathrm{m})$, $0.90(3 \mathrm{H}, \mathrm{d}, J 6.4), 1.13(3 \mathrm{H}, \mathrm{s}), 1.23(3 \mathrm{H}, \mathrm{s}), 1.36(3 \mathrm{H}, \mathrm{s}), 1.40$ $1.87(5 \mathrm{H}, \mathrm{m}), 1.90-2.16(5 \mathrm{H}, \mathrm{m}), 2.17-2.30(2 \mathrm{H}, \mathrm{m}), 2.46(1 \mathrm{H}$, ddd, $J 10.6,10.6,5.5), 2.88(1 \mathrm{H}$, br s, OH), 4.90 (1 H, ddd, $J$ $10.6,10.6,4.4), 7.15-7.19(1 \mathrm{H}, \mathrm{m})$ and $7.23-7.36(4 \mathrm{H}, \mathrm{m})$; 
$\delta_{\mathrm{C}}\left(\mathrm{CDCl}_{3}\right) 20.3(\mathrm{q}), 21.8(\mathrm{q}), 24.3(\mathrm{q}), 24.8(\mathrm{q}), 26.8(\mathrm{t}), 26.9(\mathrm{t})$, $27.1(\mathrm{t}), 31.4(\mathrm{~d}), 34.6(\mathrm{t}), 38.7(\mathrm{t}), 39.9(\mathrm{~s}), 40.7(\mathrm{t}), 49.8(\mathrm{~d}), 55.8$ (d), 73.6 (s), 77.9 (d), 125.2 (d), 125.6 (d, 2 C), 128.0 (d, 2 C), $151.4(\mathrm{~s}), 167.0(\mathrm{~s})$ and $217.4(\mathrm{~s}) ; \mathrm{m} / \mathrm{z} 386\left(\mathrm{M}^{+}, 1 \%\right)$, and 119 (100) (Found: $\mathrm{M}^{+}, 386.2610$. Calc. for $\mathrm{C}_{24} \mathrm{H}_{34} \mathrm{O}_{4}, M$, 386.2613).

5-Methyl-2-(1-methyl-1-phenylethyl)cyclohexyl 2-Hydroxy2-(2-oxocyclohexyl)propanoate 20.-2S,3S-Isomer: oil; HPLC ( $15 \%$ EtOAc in hexane) $t_{\mathrm{R}} 10.2 \mathrm{~min} ;[\alpha]_{\mathrm{D}}^{25}+30.8\left(\mathrm{CHCl}_{3}, c 5.7\right)$; $v_{\max }($ neat $) / \mathrm{cm}^{-1} 3542$ and $1721 ; \delta_{\mathrm{H}}\left(\mathrm{CDCl}_{3}\right) 0.79-1.10(2 \mathrm{H}, \mathrm{m})$, $0.86(3 \mathrm{H}, \mathrm{d}, J 6.4), 1.08(3 \mathrm{H}, \mathrm{s}), 1.21(3 \mathrm{H}, \mathrm{s}), 1.30-1.78(7 \mathrm{H}, \mathrm{m})$, $1.30(3 \mathrm{H}, \mathrm{s}), 1.79-2.18(5 \mathrm{H}, \mathrm{m}), 2.19-2.47(3 \mathrm{H}, \mathrm{m}), 3.05(1 \mathrm{H}$, br s, OH), $4.75(1 \mathrm{H}$, ddd, $J 10.6,10.6,4.3), 7.17-7.21(1 \mathrm{H}, \mathrm{m})$ and 7.25-7.38 (4 H, m); $\delta_{\mathrm{C}}\left(\mathrm{CDCl}_{3}\right) 21.7(\mathrm{q}), 22.9(\mathrm{q}), 24.7(\mathrm{q})$, $26.4(\mathrm{q}), 26.8(\mathrm{t}), 27.0(\mathrm{~d}, 2 \mathrm{C}), 27.4(\mathrm{t}), 31.2(\mathrm{~d}), 34.6(\mathrm{t}), 39.7(\mathrm{~s})$, $40.5(\mathrm{t}), 42.1(\mathrm{t}), 49.8(\mathrm{~d}), 56.1(\mathrm{~d}), 73.4(\mathrm{~s}), 76.9(\mathrm{~d}), 125.1(\mathrm{~d})$, 125.5 (d, 2 C), 127.9 (d, 2 C), $151.7(\mathrm{~s}), 175.9$ (s) and $212.4(\mathrm{~s})$; $m / z 400\left(\mathrm{M}^{+}, 3 \%\right)$ and $119(100)$ (Found: $\mathrm{M}^{+}, 400.2601$. Calc. for $\left.\mathrm{C}_{25} \mathrm{H}_{36} \mathrm{O}_{4}, M, 400.2613\right)$. $2 S, 3 S$-Isomer: solid, m.p. 113$115^{\circ} \mathrm{C}$; HPLC $(15 \%$ EtOAc in hexane $) t_{\mathrm{R}} 11.0 \mathrm{~min} ;[\alpha]_{\mathrm{D}}^{25}-25.4$ $\left(\mathrm{CHCl}_{3}, c 1.5\right) ; v_{\max }(\mathrm{KBr}) / \mathrm{cm}^{-1} 3542$ and $1725 ; \delta_{\mathrm{H}}\left(\mathrm{CDCl}_{3}\right)$ $0.77-1.07(2 \mathrm{H}, \mathrm{m}), 0.87(3 \mathrm{H}, \mathrm{d}, J 6.4), 1.21(3 \mathrm{H}, \mathrm{s}), 1.25(3 \mathrm{H}$, s), 1.37-1.83 (7 H, m), $1.40(3 \mathrm{H}, \mathrm{s}), 1.84-2.08(5 \mathrm{H}, \mathrm{m}), 2.13-$ $2.58(2 \mathrm{H}, \mathrm{m}), 2.60(1 \mathrm{H}, \mathrm{m}), 3.28(1 \mathrm{H}, \mathrm{br} \mathrm{s}, \mathrm{OH}), 4.87(1 \mathrm{H}, \mathrm{ddd}$, $J$ 10.6, 10.6, 4.3), 7.15-7.20 (1 H, m) and 7.25-7.37 (4 H, m); $\delta_{\mathrm{C}}\left(\mathrm{CDCl}_{3}\right) 21.7(\mathrm{q}), 22.9(\mathrm{q}), 24.7(\mathrm{q}), 26.4(\mathrm{q}), 26.9(\mathrm{t}), 27.4(\mathrm{t})$, $27.7(\mathrm{t}), 31.2(\mathrm{~d}), 34.5(\mathrm{t}), 34.6(\mathrm{t}), 39.7(\mathrm{~s}), 40.5(\mathrm{t}), 42.1(5), 49.8$ (d), 56.1 (d), 73.4 (s), 76.8 (d), 125.1 (d), 125.2 (d, 2 C), 127.9 (d, 2 C), 151.6 (s), 175.9 (s) and 212.4 (s); $m / z 400\left(\mathrm{M}^{+}, 1 \%\right)$ and 119 (100) (Found: $\mathrm{M}^{+}, 400.2611$. Calc. for $\mathrm{C}_{25} \mathrm{H}_{36} \mathrm{O}_{4}, M$, 400.2613).

5-Methyl-2-(1-methyl-1-phenylethyl)cyclohexyl 2-Hydroxy2-(2-oxo-5-tert-butylcyclohexyl)propanoate 21.-2S,3S-Isomer: oil; HPLC $15 \%$ EtOAc in hexane) $t_{\mathrm{R}} 4.8 \mathrm{~min}$; $[\alpha]_{\mathrm{D}}^{25}-22.3$ $\left(\mathrm{CHCl}_{3}, c 1.3\right) ; v_{\max }($ neat $) / \mathrm{cm}^{-1} 3544$ and $1721 ; \delta_{\mathrm{H}}\left(\mathrm{CDCl}_{3}\right)$ $0.77-1.10(3 \mathrm{H}, \mathrm{m}), 0.87(9 \mathrm{H}, \mathrm{s}), 0.88(3 \mathrm{H}, \mathrm{d}, J 6.4), 1.15-1.73(8$ $\mathrm{H}, \mathrm{m}), 1.26(3 \mathrm{H}, \mathrm{s}), 1.37(3 \mathrm{H}, \mathrm{s}), 1.48(3 \mathrm{H}, \mathrm{s}), 1.74-2.05(2 \mathrm{H}$, $\mathrm{m}), 2.06-2.25(2 \mathrm{H}, \mathrm{m}), 2.47(1 \mathrm{H}, \mathrm{m}), 2.57(1 \mathrm{H}, \mathrm{br} \mathrm{s}, \mathrm{OH}), 4.90$ $(1 \mathrm{H}$, ddd, $J 10.6,10.6,4.4)$ and $7.10-7.40(5 \mathrm{H}, \mathrm{m}) ; \delta_{\mathrm{C}}\left(\mathrm{CDCl}_{3}\right)$ $21.7(\mathrm{q}), 21.9$ (q), 22.6 (q), 22.9 (q), 26.0 (q), 26.9 (q), 27.3 (q), $27.5(\mathrm{t}), 27.6(\mathrm{t}), 28.1(\mathrm{~s}), 31.2(\mathrm{~d}), 32.6(\mathrm{~d}), 34.6(\mathrm{t}), 39.7(\mathrm{~s}), 40.2$ $(\mathrm{t}), 41.4(\mathrm{t}), 46.2(\mathrm{t}), 49.2(\mathrm{~d}), 55.2(\mathrm{~d}), 72.2(\mathrm{~s}), 76.9(\mathrm{~d}), 125.1(\mathrm{~d})$, $125.4(\mathrm{~d}, 2 \mathrm{C}), 128.0(\mathrm{~d}, 2 \mathrm{C}), 151.5(\mathrm{~s}), 175.7(\mathrm{~s})$ and $212.5(\mathrm{~s})$; $m / z 456\left(\mathrm{M}^{+}, 5 \%\right)$ and $119(100) .2 S, 3 S$-Isomer: oil HPLC $(15 \%$ EtOAc in hexane) $t_{\mathrm{R}} 6.0 \mathrm{~min} ;[\alpha]_{\mathrm{D}}^{25}+28.5\left(\mathrm{CHCl}_{3}<5.5\right)$; $v_{\max }($ neat $) / \mathrm{cm}^{-1} 3540$ and $1720 ; \delta_{\mathrm{H}}\left(\mathrm{CDCl}_{3}\right) 0.76-1.10(3 \mathrm{H}, \mathrm{m})$, $0.88(3 \mathrm{H}, \mathrm{d}, J 6.4), 0.97(3 \mathrm{H}, \mathrm{s}), 1.10(3 \mathrm{H}, \mathrm{s}), 1.24(3 \mathrm{H}, \mathrm{s}), 1.27-$ $1.52(8 \mathrm{H}, \mathrm{m}), 1.92(3 \mathrm{H}, \mathrm{s}), 1.99-2.21(3 \mathrm{H}, \mathrm{m}), 2.22-2.51(2 \mathrm{H}$, $\mathrm{m}), 3.04(1 \mathrm{H}$, br s, OH), $4.72(1 \mathrm{H}$, ddd, $J 10.5,10.5,4.6), 7.11-$ $7.23(1 \mathrm{H}, \mathrm{m})$ and $7.25-7.40(4 \mathrm{H}, \mathrm{m}) ; \delta_{\mathrm{C}}\left(\mathrm{CDCl}_{3}\right) 21.7(\mathrm{q}), 23.0$ (q), 26.1 (q), 26.9 (q), 27.3(q), 27.5 (q), $27.6(\mathrm{q}), 27.7(\mathrm{t}), 27.9(\mathrm{t})$, $28.1(\mathrm{~s}), 31.2(\mathrm{~d}), 32.6(\mathrm{~d}), 34.6(\mathrm{t}), 39.8(\mathrm{~s}), 40.5(\mathrm{t}), 41.3(\mathrm{t}), 46.4$ (t), 49.7 (d), 55.3 (d), 73.5 (s), 76.9 (d), 125.2 (d), 125.5 (d, 2 C), $127.9(\mathrm{~d}, 2 \mathrm{C}), 151.8(\mathrm{~s}), 175.9(\mathrm{~s})$ and $212.6(\mathrm{~s}) ; \mathrm{m} / \mathrm{z} 456\left(\mathrm{M}^{+}\right.$, $3 \%$ ) and $119(100)$ (Found: $\mathrm{M}^{+}, 456.3227$. Calc. for $\mathrm{C}_{29} \mathrm{H}_{44} \mathrm{O}_{4}$, $M, 456.3240$ ).

1-[5-Methyl-2-(1-methyl-1-phenylethyl)cyclohexyl] 4-Methyl 2-Hydroxy-3,3-dimethyl-2-phenylbutanedioate 22. $2 R$-Isomer: oil; TLC ( $5 \%$ EtOAc in hexane) $R_{\mathrm{f}} 0.20 ; v_{\max }$ (neat) $/ \mathrm{cm}^{-1} 3553$ and $1720 \mathrm{~cm}^{-1} ; \delta_{\mathrm{H}}\left(\mathrm{CDCl}_{3}\right) 0.66-0.98(3 \mathrm{H}, \mathrm{m}), 0.78(3 \mathrm{H}, \mathrm{d}, J$ 6.4), $1.14(3 \mathrm{H}, \mathrm{s}), 1.18-1.34(3 \mathrm{H}, \mathrm{m}), 1.30(3 \mathrm{H}, \mathrm{s}), 1.31(3 \mathrm{H}, \mathrm{s})$, $1.40(3 \mathrm{H}, \mathrm{s}), 1.59(3 \mathrm{H}, \mathrm{s}), 1.81-1.94(2 \mathrm{H}, \mathrm{m}), 3.77(3 \mathrm{H}, \mathrm{s}), 4.51$ $(1 \mathrm{H}, \mathrm{s}, \mathrm{OH}), 4.86(1 \mathrm{H}$, ddd, $J 10.5,10.5,4.3), 7.12-7.19(3 \mathrm{H}$, $\mathrm{m}), 7.23-7.37(5 \mathrm{H}, \mathrm{m})$ and $7.45-7.50(2 \mathrm{H}, \mathrm{m}) ; \delta_{\mathrm{C}}\left(\mathrm{CDCl}_{3}\right) 14.0$ (q), $21.7(\mathrm{q}), 22.0(\mathrm{q}), 22.6(\mathrm{q}), 22.7$ (q), $23.5(\mathrm{q}), 27.4(\mathrm{t}), 30.0$ (q), $31.3(\mathrm{~d}), 31.5(\mathrm{~s}), 34.3(\mathrm{t}), 40.4(\mathrm{~s}), 40.5(\mathrm{t}), 50.1(\mathrm{~s}), 76.9(\mathrm{~s})$, 82.6 (s), 125.3 (d), 125.8 (d, 2c), 127.3 (d, 2 C), 127.8 (d, 2 C), $128.0(\mathrm{~d}, 2 \mathrm{C}), 137.0(\mathrm{~s}), 150.5(\mathrm{~s})$ and $179.4(\mathrm{~s}) ; \mathrm{m} / \mathrm{z} 467\left(\mathrm{M}^{+}\right.$, $1 \%$ ), $207(100)$ and $119(100)$ (Found: $\mathrm{M}^{+}, 466.2711$. Calc. for $\left.\mathrm{C}_{29} \mathrm{H}_{38} \mathrm{O}_{5}, M, 466.2719\right)$.

1-[5-Methyl-2-(1-methyl-1-phenylethyl)cyclohexyl] 4-Methyl 3-Ethyl-2-hydroxy-2-phenylbutanedioate 23. $2 R, 3 R$-Isomer: oil; TLC $\left(3 \%\right.$ EtOAC in hexane) $R_{\mathrm{f}} 0.16 ; v_{\max }($ neat $) / \mathrm{cm}^{-1} 3430$ and $1730 ; \delta_{\mathrm{H}}\left(\mathrm{CDCl}_{3}\right) 0.69-1.08(3 \mathrm{H}, \mathrm{m}), 0.76(3 \mathrm{H}, \mathrm{d}, J 6.4)$, $1.00(3 \mathrm{H}, \mathrm{t}, J 7.4), 1.12(3 \mathrm{H}, \mathrm{s}), 1.17(3 \mathrm{H}, \mathrm{s}), 1.20-1.67(5$ $\mathrm{H}, \mathrm{m}), 1.84-2.00(2 \mathrm{H}, \mathrm{m}), 3.44(3 \mathrm{H}, \mathrm{s}), 3.45(1 \mathrm{H}, \mathrm{dd}, J 10.9$, 4.1), $3.72(1 \mathrm{H}, \mathrm{s}, \mathrm{OH}), 4.85$ (1 $\mathrm{H}$, ddd, $J 10.5,10.5,4.2)$, $7.16-7.35(8 \mathrm{H}, \mathrm{m})$ and $7.54-7.59(2 \mathrm{H}, \mathrm{m}) ; \delta_{\mathrm{c}}\left(\mathrm{CDCl}_{3}\right) 12.3$ (q), $21.6(\mathrm{q}), 21.8(\mathrm{t}), 24.1(\mathrm{q}), 27.2(\mathrm{t}), 29.2(\mathrm{q}), 31.2(\mathrm{~d}), 34.2$ (t), 40.1 (s), 40.8 (t), 50.1 (d), 51.5 (q), 54.5 (d), 77.2 (d), 79.9 (s), 125.1 (d), 125.2 (d, 2 C), 125.6 (d, 3 C), 127.9 (d 2 C), $128.0(\mathrm{~d}, 2 \mathrm{C}), 140.0(\mathrm{~s}), 150.3(\mathrm{~s}), 171.0(\mathrm{~s})$ and $174.4(\mathrm{~s}) ; \mathrm{m} / \mathrm{z}$ $466\left(\mathrm{M}^{+}, 30 \%\right)$ and $(100)$.

\section{Acknowledgements}

We thank the National Science Council for financial support (Grant NSC82-0208-M002-031).

\section{References}

1 H.-U. Blaser, Chem. Rev., 1992, 92, 935.

2 M. Ihara, M. Takahashi, N. Taniguchi, K. Yasui, K. Fukumoto and T. Kametani, J. Chem. Soc., Perkin Trans. 1, 1989, 897; M. Ihara, M. Takahashi, H. Niitsuma, N. Taniguchi, K. Yasui and K. Fukumoto, J. Org. Chem., 1989, 54, 5413.

3 W. Oppolzer and H. J. Loher, Helv. Chim. Acta, 1981, 64, 2808; E. J. Corey and R. T. Peterson, Tetrahedron Lett., 1985, 26, 5025 .

4 E. J. Corey and H. E. Ensley, J. Am. Chem. Soc., 1975, 97, 6908; W. Oppolzer, M. Kurth, D. Reichlin, C. Chapuis, M. Mohnhaupt and F. Moffatt, Helv. Chim. Acta, 1981, 64, 2802.

5 O. Takahashi, K. Mikami and T. Nakai, Chem. Lett., 1987, 69.

6 R. Vaßen, J. Runsink and H.-D. Scharf, Chem. Ber., 1986, 119, 3492; D. Crich and J. W. Davies, Tetrahedron Lett., 1991, 32, 27; D. Crich and L. Quintero, Chem. Rev., 1989, 89, 1413; M.-Y. Chen, J.-M. Fang, Y.-M. Tsai and R.-L. Yeh, J. Chem. Soc., Chem. Commun., 1991, 1604.

7 J. K. Whitesell, D. Deyo and A. Bhattacharya, J. Chem. Soc., Chem. Commun., 1983, 802.

8 J. K. Whitesell, A. Bhattacharya and K. Henke, J. Chem. Soc., Chem Commun., 1982, 988.

9 Y. Yamamoto, K. Maruyama, H. Yatagai, Y. Ishihara and N. Maeda, Tetrahedron, 1984, 40, 2239; P. Grossen, P. Herold, P. Mohr and C. Tamm, Helv. Chim. Acta, 1984, 67, 1625.

$10 \mathrm{~J}$. Hubscher and R. Barner, Helv. Chim. Acta, 1990, 73, 1068

11 M.-Y. Chen. and J.-M. Fang, J. Org. Chem., 1992, 57, 2937.

12 J. K. Whitesell, A. Bhattacharya, D. A. Aguilar and K. Henke, J. Chem. Soc., Chem. Commun., 1982, 989; J. K. Whitesell, K. Nabona and D. Deyo, J. Org. Chem., 1989, 54, 2258. Although (-)-8phenylmenthyl glyoxylates undergo ene reactions with hex-1-ene in the presence of $\mathrm{SnCl}_{4},(-)-8$-phenylmenthyl pyruvates undergo selfcondensation under similar conditions.

13 A. Solladie-Cavallo and N. Khiar, Tetrahedron Lett., 1988, 29, 2189.

14 M. Weuthen, H.-D. Scharf, J. Runsink and R. Vaßen, Chem. Ber., $1988,121,971$

15 M. T. Reetz and A. Jung, J. Am. Chem. Soc., 1983, 105, 4833; S. Kobayashi and I. Hachiya, J. Org. Chem., 1992, 57, 1324.

16 H. Moorlag, R. M. Kellogg, M. Kloosterman, B. Kaptein, J. Kamphuis and H. E. Schoemaker, J. Org. Chem., 1990, 55, 5878; G. Frater, U. Müller and W. Günther, Tetrahedron Lett., 1981, 22, 4221.

17 I. Ojima, Y. Miyazawa and M. Kumagai, J. Chem. Soc., Chem. Commun., 1976, 927; I. Ojima, K. Yoshida and S.-I. Inaba, Chem. Lett., 1977, 429.

18 Y. Yamamoto, K. Maruyama, T. Komatsu and W. Ito, J. Org. Chem., 1986, 51, 886; J.-M. Fang and B.-C. Hong, J. Org. Chem., 1987, 52, 
3162; Z. Wang, X.-J. Meng and G. W. Kabalka, Tetrahedron Lett., 1991, 32, 5677.

19 C.-C. Yang and J.-M. Fang, J. Chem. Soc., Perkin Trans. 1, 1992, 3085.

20 K. Matsunoto and K. Harada, J. Org. Chem., 1966, 31, 1956.

21 O. Ort, Org. Synth., 1986, 65, 203.

22 H. O. House, L. J. Ozuba, M. Gall and H. D. Olmstead, J. Org.
Chem., 1969, 34, 2324; R. E. Ireland, R. H. Mueller and A. K. Willard, J. Am. Chem. Soc., 1976, 98, 2868

Paper 3/02084C Received 13th April 1993 Accepted 28th April 1993 